\title{
Chunjie Zhang: Transculturality and German Discourse in the Age of European Colonialism
}

Evanston: Northwestern University Press 2017 - ISBN 978-0-81013-477-5 - 34,95 \$

$\mathrm{Zu}$ den scheinbar unverrückbaren und deshalb nur selten hinterfragten Gewissheiten im Bereich der postkolonialen Literaturwissenschaft gehört die Behauptung, in den Texten europäischer Autoren sorge eine durchgängig eurozentristische Perspektive dafür, dass über Begegnungen mit nichteuropäischen Kulturen ausschließlich das wiedergegeben werden könne, was die Stereotypen des jeweiligen Verfassers zuließen, ja es werde immer und überall nur das gesehen, was das eigene Weltbild zu bestätigen in besonderem Maße geeignet sei. Die Allgemeingültigkeit eben dieser Behauptung versucht Chunjie Zhang in ihrer Aufsatzsammlung Transculturality and German Discourse zu erschüttern, indem sie proklamiert, nichteuropäische Vorstellungen konstituierten die Texte der von ihr untersuchten Autoren (Georg Forster, Adelbert von Chamisso, Joachim Heinrich Campe, August von Kotzebue, Johann Gottfried Herder, Immanuel Kant) zu einem ganz erheblichen Teil mit (vgl. 3, 10, 16f., 43, 46, 58, 64 u.ö.).

Vor dem Hintergrund der eingangs erwähnten Einseitigkeit postkolonialer Lektüren stellt Zhangs Ansatz damit eine wichtige Neuperspektivierung dar, die entscheidend $\mathrm{zu}$ einer ausgewogeneren Betrachtung deutschsprachiger Texte über das beitragen könnte, was Zhang in ihrer Einleitung als Transkulturalität definiert (vgl. 1of.). Als schwierig erweist sich allerdings bereits im Rahmen dieser Definition, dass zwar Abgrenzungen gegen konkurrierende Begriffe wie Transnationalismus, Multikulturalität oder Interkulturalität vorgenommen (vgl. 10), letztlich aber zur inhaltlichen Füllung der Transkulturalität nicht unproblematische Metaphern herangezogen werden, etwa wenn Zhang dem »non-European knowledge« in Bezug auf die Konstitution der untersuchten Texte »an active participation « zuweist oder einfach von einer »non-European agency« (11) spricht.

So wird nicht immer ganz klar, ob Zhang auf den Nachweis der - letztlich unbestreitbaren - Tatsache einer Beeinflussung Forsters, Chamissos, Campes, Kotzebues, Herders und Kants durch Wissensbestände um außereuropäische Gegebenheiten abzielt oder ob sie tatsächlich demonstrieren will (und kann), dass ein spezifisch nichteuropäisches, vom Wissenschaftsverständnis der Aufklärung fundamental verschiedenes Denkmodell an der Konstitution der jeweiligen Texte aktiv - und als selbständige Entität erkennbar - beteiligt ist: Ersteres wäre als These vergleichsweise banal (im Detail aber natürlich dennoch von Interesse für die Literaturwissenschaft), Letzteres eine mittlere Sensation zumal für Ethnologie und Anthropologie.

Leider bleiben die Nachweise Zhangs für ihre immer wieder mantraartig wiederholte Kernaussage häufig der bereits für die Definition konstatierten Metaphorik verpflichtet; konkret greifbar erscheint ihr Ansatz am ehesten noch in der Analyse eines 
von Chamisso mit der Unterstützung seines Gewährsmannes Kadu erstellten Vokabulars, das in Zhangs Worten als »coconstructed by him [sc. Chamisso] and Kadu« (53) aufzufassen sei. Nun stellt Chamissos Aussage über Kadu, dieser sei für ihn zu einer »wissenschaftlichen Autorität « geworden (Chamisso, zit. n. 194, Endnote 16 - deutsche Originalzitate finden sich bei Zhang grundsätzlich nur in den Endnoten), tatsächlich die eindeutige Präsentation eines Verhältnisses zwischen gleichberechtigten Partnern und Koautoren dar; gerade hier könnte und müsste der Nachweis einer aktiven Rolle der Nichteuropäer bei der Konstitution des Wissens in der europäischen Aufklärung ansetzen.

Selbst im Chamisso-Kapitel allerdings bleiben Zhangs Ausführungen zu Kadus Mittlerrolle (vgl. 45) äußerst vage: Zunächst wird auf Kadus eigene Reisen hingewiesen, um diesem einen Cook/Forster und Kotzebue/Chamisso vergleichbaren Entdeckergestus zuzuschreiben (vgl. 46). Dann wird der wohlbekannte Unterschied zwischen den eher objektiven Betrachtun gen und Ansichten und der eher subjektiven Reise um die Welt dargestellt (vgl. 47-50), die französisch-deutsch-russische Zwischenstellung des Forschers Chamisso analysiert (vgl. 50-52), bevor erneut Kadu in den Mittelpunkt des Interesses rückt: Zhang referiert Chamissos Beobachtungen zur Assimilation Kadus an seine europäische Umgebung während der Reise auf der Rurik (vgl. 53f.), bevor sie aufzählt, welche Informationen Kadu den Expeditionsteilnehmern und insbesondere Chamisso vermittelt (vgl. 54-59).

Eine besondere Rolle spielen dabei die den Europäern überlegenen
Navigationskünste der Insulaner, die sich für einen Wissenstransfer so geradezu anbieten - auf eine entsprechende Spurensuche in Chamissos und/oder Kotzebues Texten verzichtet Zhang dann aber bedauerlicherweise vollständig. Stattdessen werden Chamissos Wunsch, sich tätowieren $\mathrm{zu}$ lassen, sowie seine Kritik an der christlichen Mission im Pazifik als Zeichen seiner besonderen Verbundenheit mit den Insulanern hervorgehoben (vgl 59-62). Dieselbe Funktion erfüllen die Hinweise auf Chamissos Protest gegen die abwertende Bezeichnung der Insulaner als Wilde (vgl. 56) und gegen den Kadu vom Verleger Maltebrun unterstellten Kannibalismus (vgl. 63). All das zeigt Chamissos positive Einstellung dem Fremden gegenüber, trägt aber zum Nachweis der »non-European agency« bei der Herstellung der untersuchten Texte so gut wie nichts bei.

Noch problematischer gestaltet sich das Aufspüren dieser aktiven Beteiligung nichteuropäischer Agenten an der Erstellung deutscher Texte in den übrigen Kapiteln. Die grundsätzliche Verwechslung positiver Äußerungen über Ozeanien und seine Bewohner mit einer messbaren Beeinflussung durch dieselben bei der Textproduktion dominiert das gesamte Forster-Kapitel. Dieses rückt Forsters Verständnis für einen auf Tahiti desertierten Matrosen, für den Kannibalismus der Insulaner sowie für deren Recht auf Selbstverteidigung in den Mittelpunkt, legt dabei aber - eigentlich im strikten Widerspruch zu Zhangs Grundthese - eher stringent und nachvollziehbar offen, in welchem Ausmaß Forsters europäische Traditionen und Kategorien seine Ur- 
teilsfindung bestimmen (vgl. z.B. den berechtigten Hinweis auf Ovids Bearbeitung des Mythos von Philemon und Baucis als Deutungsmuster für die ozeanische Gastfreundschaft, 33).

Kategorial von der Analyse der Reiseberichte Forsters und Chamissos unterschieden sind dann die vier folgenden Kapitel, denn Campe, Kotzebue, Herder und Kant gelangen ja durch Reiseberichte wie denjenigen Forsters überhaupt erst in - damit stets vermittelten - Kontakt zur außereuropäischen Realität. Hier kann keine (direkte) »non-European agency« postuliert werden, was Zhang konsequenterweise auch nicht tut. Stattdessen zeigt sie an vier Stücken Kotzebues (La Peyrouse, Bruder Moritz, Die Spanier in Peru, Rollas Tod), wie diese als Melodramen nicht nur bewusst gegen das bürgerliche Trauerspiel sowie die Tragödientheorien der Weimarer Klassik konzipiert sind, sondern die außereuropäischen Stoffe bewusst zur Herbeiführung eines Happy Ends funktionalisieren.

Dabei würden in La Peyrouse eine freizügige Sexualmoral, in Bruder Moritz die Überwindung rassistischer und ständischer Vorurteile, in den Spaniern in Peru ein Triumph der Liebe und in Rollas Tod ein altruistisches Heldentum gefeiert, die so in Europa jeweils nicht $\mathrm{zu}$ finden seien und deshalb nur in Übersee ein glückliches Ende der Handlung ermöglichen könnten. Dass Zhang diese von Kotzebue den außereuropäischen Gesellschaften zugeschriebenen Ideale nicht als typisch europäische Wunschvorstellungen erkennt, ist nun durchaus bedenklich. Denn Kotzebue praktiziert hier schließlich und endlich nichts anderes als das, was bereits die antike
Ethnographie, besonders prominent durch die Germania des Tacitus vertreten, durchgängig als Aufbauprinzip ihrer Texte verwendet hat: Er schreibt den sedlen Wilden all die positiven Eigenschaften zu, die die eigene, nach Ansicht des Dichters heillos korrumpierte Gesellschaft angeblich längst verloren hat.

Allen durchaus überzeugenden Überlegungen zur Gattungsgeschichte des Melodrams zum Trotz scheitert Zhangs Analyse der vier Dramen daher an grundsätzlichen Missverständnissen: Wichtiger als die postkoloniale Theorie sowie deren Weiterentwicklung durch Zhang wäre für das Verständnis von Kotzebues Gesellschaftskritik - deren biographische Grundlagen zu Recht betont werden (vgl. 9of.) - ein Blick auf die aufklärerischen Nachfolger des Tacitus, beispielsweise auf Montesquieus berühmte Lettres persanes, gewesen. Deren kommunikative Grundstruktur hätte in ihrer Parallelität zur dramatischen Technik Kotzebues besonders deutlich zeigen können, wie akzidentiell letztlich außereuropäische Realien für die Intention eines Autors sind, dem es darum zu tun ist, innereuropäische Missstände durch einen konstruierten Blick von außen aufzudecken.

Campe wiederum spielt für Zhang ungefähr dieselbe Rolle wie Forster in Bezug zu Chamisso: Auch er bildet durch seine stärkere Verhaftung im Denken der europäischen Aufklärung die (schwarze) Folie, vor der sich Kotzebues vorgebliche Offenheit außereuropäischen Einflüssen gegenüber umso strahlender abheben soll. So bleibt auch bei Campe von der angeblich aktiven Rolle dieser Einflüsse letztlich nur der Beitrag übrig, 
den der Freundschaftsbund mit Freitag zur Persönlichkeitsbildung Krusoe Robinsons leistet. Dass diese - anders als von Campe als Ideal seines Erziehungsprogramms formuliert - mit einer zunehmenden Emotionalisierung einhergeht (vgl. 83f.), wird denn auch lediglich festgestellt, mit der folgenden Wiederholung der Grundthese aber in keinerlei logischen oder auch nur argumentativen Zusammenhang gebracht (vgl. 84f.), obgleich sich hier möglicherweise tatsächlich Gegenpositionen zur aufklärerischen Rationalität und so vielleicht auch deren Herkunft aus außereuropäischen Denkmodellen hätten belegen lassen.

Für die abschließende Betrachtung der beiden Philosophen Herder und Kant ändert Zhang das bislang verwendete Aufbauschema: Nun werden im umfangreichsten Kapitel des Buches - gegen Chronologie und geistesgeschichtliche Entwicklung zunächst Schriften aus Früh- und Spätwerk Herders, erst im Anschluss daran aber die aus Notizen und studentischen Mitschriften rekonstruierte Physikalische Geographie Kants betrachtet. Für Herder untersucht Zhang dabei die Spannungen zwischen dessen kulturellem Relativismus und einer teleologisch ausgerichteten Universalgeschichte, die diesem Relativismus insofern widerspreche, als die Entwicklungsstufe Nordeuropas derjenigen anderer Erdteile hierarchisch übergeordnet werde. Obgleich Zhang sich redlich darum bemüht, bei dieser Gegenüberstellung nicht in ein typisch postkoloniales Gut-Böse-Schema zu verfallen, leidet auch dieses Kapitel darunter, dass die Grundthese der Monographie für die an Herders Philosophie herangetragenen Fragen kaum eine Rolle spielt.
Leider gilt beinahe dasselbe letztlich auch für das Kapitel zur Physikalischen Geographie, obgleich hier ein Ansatz entwickelt wird, der fast eher noch als selbst das Chamisso-Kapitel dazu geeignet scheint, der ominösen »non-European agency « endlich auf die Spur zu kommen. Ausgehend von der Entstehungsgeschichte des Textes nämlich kann Zhang diesen überzeugend als »an educational project, an event in intellectual history« definieren, das »a transcultural consciousness of global interconnectedness in the space-time around 1800 « (169) ermöglicht habe. Dabei stelle weniger Kants Überzeugung eines Vorrangs der Geographie vor der Geschichte, der das Dilemma Herders auflösen könne (vgl. 168), als vielmehr die unabgeschlossene Offenheit des Textes die Grundvoraussetzung für eine konstante Beeinflussung desselben durch »non-European knowledge « in Form einer »active participation « dar.

Wer im 18. Jahrhundert über vierzig Jahre lang eine Vorlesung zur Geographie hält, ist zweifellos in ganz außergewöhnlichem Maße dazu gezwungen, die eigenen Unterlagen immer wieder dem rasanten Wissenszuwachs anzupassen (vgl. 17lf.). Diesen Veränderungen im offenen Text der Physikalischen Geographie nachzuspüren, wäre daher insbesondere deshalb ein lohnendes Unterfangen. Denn hier könnte über Kants Quellen, deren Analyse aus zweiter Hand Zhang jedoch leider lediglich summarisch referiert (164f.), tatsächlich ein außereuropäischer Einfluss nachgewiesen werden: Dazu freilich müssten dann zunächst - ähnlich wie insbesondere für Chamisso und seinen Gewährsmann Kadu - konkrete nichteuropä- 
ische Vorstellungen in den Reiseberichten europäischer Verfasser isoliert und deren in irgendeiner Weise strukturell relevant gewordene Reproduktion in der Physikalischen Geographie herausgearbeitet werden. Doch hier, wo die eigentliche philologische Arbeit beginnen müsste, lenkt Zhang bedauerlicherweise zur für ihre These vollkommen irrelevanten Frage nach Kants Rassismus über (vgl. 166).

Die an dieser Stelle formulierte Kritik am uneingelösten Versprechen einer nur formulierten, aber nicht im Detail nachgewiesenen Grundthese ändert freilich nichts daran, dass Zhang ein ungemein informatives und inspirierendes Buch geschrieben oder vielmehr aus den beinahe sämtlich bereits vorab publizierten Aufsätzen kompiliert - hat. Durchgängig werden die untersuchten Werke überzeugend in ihre historischen Kontexte und Diskurse eingeordnet, immer wieder beleuchtet Zhang instruktiv gewählte Textstellen unter ebenso überraschenden wie aufschlussreichen Gesichtspunkten: Forsters Vermittlung zwischen fremdartigem Eindruck und europäischen Denkmustern, Chamissos engagiertes Plädoyer für die wissenschaftliche Qualifikation seines
Freundes Kadu, Campes aufklärerische Kritik der Empfindsamkeit und sein präkolonialer Pragmatismus, Kotzebues in das Genre des Melodrams verpackte Gesellschaftskritik, Herders spannungsreicher Weg vom Relativismus zu einem universalen Historismus und Kants Betonung der Bedeutung geographischer Kenntnisse für das aufklärerische Projekt werden ebenso differenziert wie übersichtlich und nachvollziehbar dargestellt.

Dass ein roter Faden der ausführlichen Einleitung und den (allerdings äußerst knapp gehaltenen) Überleitungen zum Trotz am ehesten noch im letzten, offenbar eigens für die vorliegende Publikation verfassten Kapitel greifbar, ansonsten aber häufig vermisst wird, tut einer stets anregenden Lektüre keinen Abbruch im Gegenteil: Gerade der Verzicht auf eine lückenlose Einpassung aller untersuchten Elemente in das Gerüst einer einzigen Grundthese ermöglicht die vielen ungewöhnlichen Perspektiven, die Zhangs Abhandlung zu einer wirklichen Bereicherung der Forster-, Chamisso-, Campe-, Kotzebue-, Herder- und Kant-Forschung machen.

Heiko Ullrich

\section{Benoît Ellerbach: L'Arabie contée aux Allemands: Fictions interculturelles chez Rafik Schami}

Würzburg: Königshausen \& Neumann 2018 - ISBN 978-3826064845 - 49,80€

In seinem 2017 erschienenen Selbstporträt Ich wollte nur Geschichten erzählen: Mosaik der Fremde stellt der aus Syrien stammende Schriftsteller und Erzähler Rafik Schami nüchtern fest: »Ohne mein Exil in Deutschland gäbe es meine Romane und Geschich- ten nicht « (Schami 2017: 162). Zweifelsohne zählt Schami heute (nicht zuletzt auch aufgrund des jüngsten Kapitels syrischer Einwanderung nach Deutschland) zu den bekanntesten Stimmen einer inzwischen bereits über vierzig Jahre währenden Traditi- 